\title{
Twitter as a Corpus for Sentiment Analysis and Opinion Mining
}

\author{
Vaibhavi N Patodkar ${ }^{1}$, Sheikh I.R. ${ }^{2}$ \\ Student, Comp Dept, S.N.D College of Engg \& Research Center, Babhulgaon, Yeola, Nashik, India ${ }^{1}$ \\ Asst. Professor, Comp Dept, S.N.D College of Engg \& Research Center, Babhulgaon, Yeola, Nashik, India ${ }^{2}$
}

\begin{abstract}
Sentiment Analysis (SA) and summarization has recently become the focus of many researchers, because analysis of online text is beneficial and demanded in many different applications. One such application is productbased sentiment summarization of multi-documents with the purpose of informing users about pros and cons of various products. This paper introduces a novel solution to target-oriented sentiment summarization and SA of short informal texts with a main focus on Twitter posts known as "tweets". We compare different algorithms and methods for SA polarity detection and sentiment summarization. We show that our hybrid polarity detection system not only outperforms the unigram state-of-the-art baseline, but also could be an advantage over other methods when used as a part of a sentiment summarization system. Additionally, we illustrate that our SA and summarization system exhibits a high performance with various useful functionalities and features. Sentiment classification aims to automatically predict sentiment polarity (e.g., positive or negative) of users publishing sentiment data (e.g., reviews, blogs). Although traditional classification algorithms can be used to train sentiment classifiers from manually labeled text data, the labeling work can be time-consuming and ex-pensive. Meanwhile, users often use some different words when they express sentiment in different domains. If we directly apply a classifier trained in one domain to other domains, the performance will be very low due to the differences between these domains. In this work, we develop a general solution to sentiment classification when we do not have any labels in a target domain but have some labeled data in a different domain, regarded as source domain.
\end{abstract}

Keywords: Sentiment analysis, label data, sentiment polarity, sentiment classification.

\section{INTRODUCTION}

With the increasing popularity of social networking, whether a given piece of text is subjective or objective and blogging and micro-blogging websites, every day a huge if it is subjective, then whether it is negative or positive.

amount of informal subjective text statements are made available online. The information captured from these texts, could be employed for scientific surveys from a social or political perspective. Companies and product owners who aim to ameliorate their products/services may strongly benefit from the rich feedback. On the other hand, customers could also learn about positivity or negativity of different features of products/services according to users' opinions, to make an educated purchase. Furthermore, applications like rating movies based on online movie reviews could not emerge without making use of these data. "Sentiment Analysis On Twitter Data" is increasing popularity of social networking and Sentiment Analysis (SA) is one of the most widely studied applications of Natural Language Processing (NLP) and Machine Learning (ML). This field has grown tremendously with the advent of the Web 2.0. The Internet has provided a platform for people to express their views, emotions and sentiments towards products, people and life in general. Thus, the Internet is now a vast resource of opinion rich textual data. The goal of Sentiment Analysis is to harness this data in order to obtain important information regarding public opinion, that would help make smarter business decisions, political campaigns and better product consumption. Sentiment Analysis focuses on identifying

\section{OBJECTIVE}

- The objective of this project is to show how sentimental analysis can help improve the user experience over a social network or system interface.

- The learning algorithm will learn what our emotions are from statistical data then perform sentiment analysis.

- Our main objective is also maintain accuracy in the final result.

- The main goal of such a sentiment analysis is to discover how the audience perceives the television show. The Twitter data that is collected will be classified into two categories; positive or negative. An analysis will then be performed on the classified data to investigate what percentage of the audience sample falls into each category.

- Particular emphasis is placed on evaluating different machine learning algorithms for the task of twitter sentiment analysis.

\section{LITERATURE SURVEY}

I] Sentiment classification aims to automatically predict sentiment polarity (e.g., positive or negative) of users 
Vol. 5, Issue 12, December 2016

publishing sentiment data (e.g., reviews, blogs). Although the main feature,some classification methods have been traditional classification al-gorithms can be used to train proposed :Navie Bayes,Support Vector Machine,Ksentiment classifiers from manuallylabeled text data, the Nearest Neighbours,etcaccording to(Go et al,2009),it is labeling work can be time-consuming and ex-pensive. not clear which of these classification strategies is the Meanwhile, users often use some different words when more appropriate to perform sentiment analysis.

they express sentiment in different domains. If we directly We decided to use a classification strategy based on Naive apply a classifier trained in one domain to other domains, Bayes(NB)because it is a simple and intuitive method the performancewill be very low due to the differences whose performance is similar to other approaches. NB between these domains. In this work, we develop a general solution to sentiment classi-fication when we do not have any labels in a target domain but have some labeled data in a different domain, regarded as source domain

II] A sentiment classification method that is applicable when we do not have any labeled data for a target domain but have some labeled data for multiple other domains, des-ignated as the source domains. We automat-ically create a sentiment sensitive thesaurus using both labeled and unlabeled data from multiple source domains to find the associa-tion between words that express similar sentiments in different domains. The created the-saurus is then used to expand feature vectors to train a binary classifier. Unlike previous cross-domain sentiment classification meth-ods, our method can efficiently learn from multiple source domains. Our method significantly outperforms numerous baselines and returns results that are better than or com-parable to previous cross-domain sentiment classification methods on a benchmark dataset containing Amazon user reviews for different types of products.

III] Merchants selling products on the Web often ask their customers to review the products that they have purchased and the associated services. As e-commerce is becoming more and more popular, the number of customer reviews that a product receives grows rapidly. For a popular product, the number of reviews can be in hundreds or even thousands. This makes it difficult for a potential customer to read them to make an informed decision on whether to purchase the product. It also makes it difficult for the manufacturer of the product to keep track and to manage customer opinions. For the manufacturer, there are additional difficulties because many merchant sites may sell the same product and the manufacturer normally produces many kinds of products. In this research, we aim to mine and to summarize all the customer reviews of a product. This summarization task is different from traditional text summarization because we only mine the features of the product on which the customers have expressed their opinions and whether the opinions are positive or negative. We do not summarize the reviews by selecting a subset or rewrite some of the original sentences from the reviews to capture the main points as in the classic text summarization.

\section{RELATED WORK}

Most of the algorithm for sentiment analysis are based on a classifier trained using a collection of annotated text data.Before training, data is pre-processed so as to extract

\section{PROPOSED SYSTEM}

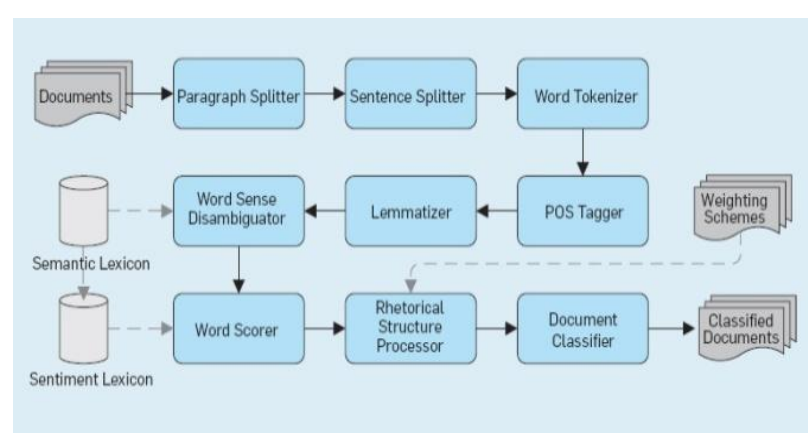

Fig 1.steps in sentiment classification

1. Paragraph spilter-It is a tool which given a text as input and it output the identified paragraph surrounded bt tages. 2.Sentence spilter- It can spilt the sentence but that was not easy to spilt its very hard to finding out puncton mark. 3.Word toknizer- Tokenization is the process of breaking stream of text up into words, phrases, symbol or other meaningful element called token

4.Word sencedisambigutor -Is a computational linguistics, word-sence ambigutation is open problem of natural language processing and ontology wsd is identifying which sence of a word is used in a sentence, when word has multipal meaning.

5.Pos tagger- Part of speech is apiece of software that reads text in some language and aassign part of speech to each word.

\section{ARCHITECTURE DIAGRAM}

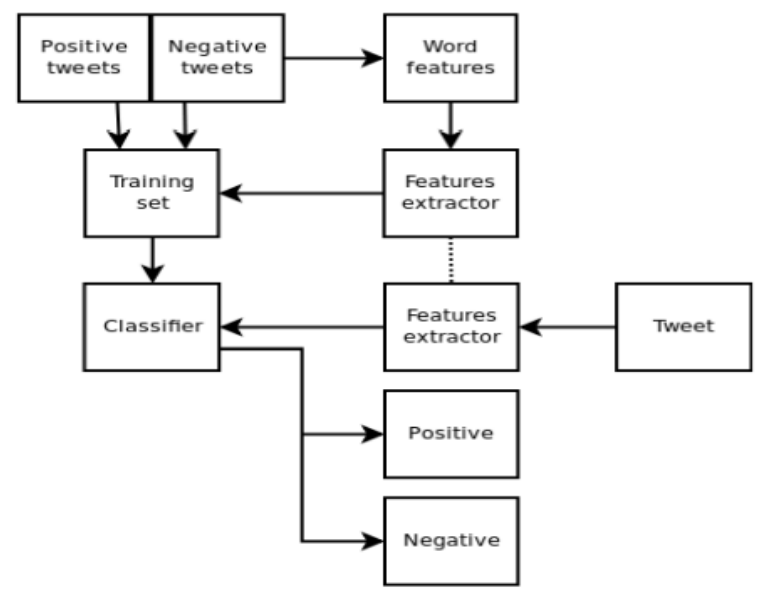

Fig. 2. Architecture diagram sentiment analysis 
Vol. 5, Issue 12, December 2016

\section{ALGORITHM MATHEMATICAL FORMULATION}

The Naive Bayesian classifier is based on Bayes' theorem with independence assumptions between predictors. A Naive Bayesian model is easy to build, with no complicated iterative parameter estimation which makes it particularly useful for very large datasets. Despite its simplicity, the Naive Bayesian classifier often does surprisingly well and is widely used because it often outperforms more sophisticated classification methods.

\section{Algorithm}

Bayes theorem provides a way of calculating the posterior probability, $\mathrm{P}(\mathrm{c} \mid \mathrm{x})$, from $\mathrm{P}(\mathrm{c}), \mathrm{P}(\mathrm{x})$, and $\mathrm{P}(\mathrm{x} \mid \mathrm{c})$. Naive Bayes classifier assume that the effect of the value of a predictor ( $\mathrm{x}$ ) on a given class (c) is independent of the values of other predictors. This assumption is called class conditional independence.

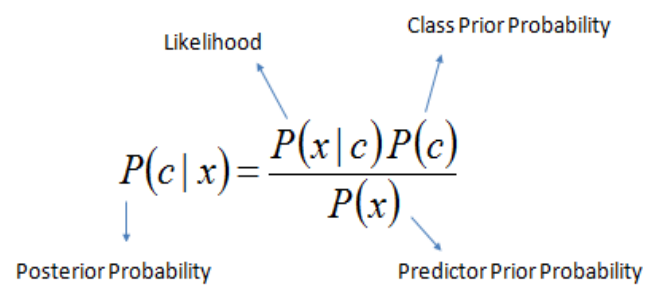

$$
P(c \mid \mathrm{X})=P\left(x_{1} \mid c\right) \times P\left(x_{2} \mid c\right) \times \cdots \times P\left(x_{n} \mid c\right) \times P(c)
$$

Above,

- $\mathrm{P}(\mathrm{c} \mid \mathrm{x})$ is the posterior probability of class (c, target) given predictor ( $\mathrm{x}$, attributes).

- $P(c)$ is the prior probability of class.

- $\mathrm{P}(\mathrm{x} \mid \mathrm{c})$ is the likelihood which is the probability of predictor given class.

- $\mathrm{P}(\mathrm{x})$ is the prior probability of predictor.

\section{CONCLUSION}

To conclude, this report has illustrated that an effective sentiment analysis can be performed on a television program by collecting a sample audience opinion from Twitter. Throughout the duration of this project many different data analysis tools were employed to collect, clean and mine sentiment from the dataset. Such an analysis could provide valuable feedback to producers and help them to spot a negative turn in viewer's perception of their show. Discovering negative trends early on can allow them to make educated decisions on how to target specific aspects of their show in order to increase its audience's satisfaction. It is apparent from this study that the machine learning classifier used has a major effect on the overall accuracy of the analysis. Commonly used algorithms for text classification were examined such as Naïve Bayes, Decision Tree, Support Vector Machine, and Random Forests. Through the evaluation of different algorithms, it was found that out of the models examined the Random
Forest algorithm using twenty random trees had the highest performance on this dataset.

\section{REFERENCES}

[1] A.Pak and P. Paroubek. ", ". In Proceedings of the Seventh Conference on International Language Resources and Evaluation, 2010, pp.1320-1326

[2] R. Parikh and M. Movassate, "Sentiment Analysis of UserGeneratedTwitter Updates using Various Classi_cation Techniques",CS224N Final Report, 2009

[3] Go, R. Bhayani, L.Huang. "Twitter Sentiment ClassificationUsing Distant Supervision". Stanford University, Technical Paper,2009

[4] L. Barbosa, J. Feng. "Robust Sentiment Detection on Twitterfrom Biased and Noisy Data". COLING 2010: Poster Volume,pp. 36-44.

[5] Bifet and E. Frank, "Sentiment Knowledge Discovery inTwitter Streaming Data", In Proceedings of the 13th InternationalConference on Discovery Science, Berlin, Germany: Springer,2010, pp. 1-15.

[6] Agarwal, B. Xie, I. Vovsha, O. Rambow, R. Passonneau, "Sentiment Analysis of Twitter Data", In Proceedings of the ACL 2011Workshop on Languages in Social Media,2011, pp. 30-38

[7] Dmitry Davidov, Ari Rappoport." Enhanced Sentiment Learning Using Twitter Hashtags and Smileys". Coling 2010: Poster Volumepages 241\{249, Beijing, August 2010

[8] Po-Wei Liang, Bi-Ru Dai, "Opinion Mining on Social MediaData", IEEE 14th International Conference on Mobile Data Management,Milan, Italy, June 3 - 6, 2013, pp 91-96, ISBN: 978-1-494673-6068-5, http://doi.ieeecomputersociety.org/ 10.1109/MDM.2013.

[9] Pablo Gamallo, Marcos Garcia, "Citius: A Naive-Bayes Strategyfor Sentiment Analysis on English Tweets", 8th InternationalWorkshop on Semantic Evaluation (SemEval 2014), Dublin, Ireland,Aug 23-24 2014, pp 171-175.

[10] Neethu M,S and Rajashree R," Sentiment Analysis in Twitter using Machine Learning Techniques" 4th ICCCNT 2013, at Tiruchengode, India. IEEE - 31661

[11] P. D. Turney, "Thumbs up or thumbs down?: semantic orientation applied to unsupervised classification of reviews," in Proceedings of the 40th annual meeting on association for computational linguistics, pp. 417-424, Association for Computational Linguistics, 2002. 